\title{
Xenorhabdus khoisanae SB10 produces Lys- rich PAX lipopeptides and a Xenocoumacin in its antimicrobial complex
}

\author{
J. Dreyer ${ }^{1}$, M. Rautenbach ${ }^{2 *}$, E. Booysen ${ }^{1}$, A. D. van Staden ${ }^{1}$, S. M. Deane ${ }^{1}$ and L. M. T. Dicks ${ }^{1 *}$ (D)
}

\begin{abstract}
Background: Xenorhabdus spp. live in close symbiosis with nematodes of the Steinernema genus. Steinernema nematodes infect an insect larva and release their symbionts into the haemocoel of the insect. Once released into the haemocoel, the bacteria produce bioactive compounds to create a semi-exclusive environment by inhibiting the growth of bacteria, yeasts and molds. The antimicrobial compounds thus far identified are xenocoumacins, xenortides, xenorhabdins, indole derivatives, xenoamicins, bicornutin and a number of antimicrobial peptides. The latter may be linear peptides such as the bacteriocins xenocin and xenorhabdicin, rhabdopeptides and cabanillasin, or cyclic, such as PAX lipopeptides, taxlllaids, xenobactin and szentiamide. Thus far, production of antimicrobial compounds have been reported for Xenorhabdus nematophila, Xenorhabdus budapestensis, Xenorhabdus cabanillasii, Xenorhabdus kozodoii, Xenorhabdus szentirmaii, Xenorhabdus doucetiae, Xenorhabdus mauleonii, Xenorhabdus indica and Xenorhabdus bovienii. Here we describe, for the first time, PAX lipopeptides and xenocoumacin 2 produced by Xenorhabdus khoisanae. These compounds were identified using ultraperformance liquid chromatography, linked to high resolution electrospray ionisation mass spectrometry and tandem mass spectrometry.
\end{abstract}

Results: Cell-free supernatants of X. khoisanae SB10 were heat stable and active against Bacillus subtilis subsp. subtilis, Escherichia coli and Candida albicans. Five lysine-rich lipopeptides from the PAX group were identified in HPLC fractions, with PAX 1' and PAX7 present in the highest concentrations. Three novel PAX7 peptides with putative enoyl modifications and two linear analogues of PAX1' were also detected. A small antibiotic compound, yellow in colour and $\lambda_{\max }$ of $314 \mathrm{~nm}$, was recovered from the HPLC fractions and identified as xenocoumacin 2 . The PAX lipopeptides and xenocoumacin 2 correlated with the genes and gene clusters in the genome of $X$. khoisanae SB10.

Conclusion: With UPLC-MS and MS analyses of compounds in the antimicrobial complex of X. khoisanae SB10, a number of PAX peptides and a xenocoumacin were identified. The combination of pure PAX1' peptide with xenocoumacin 2 resulted in high antimicrobial activity. Many of the fractions did, however, contain labile compounds and some fractions were difficult to resolve. It is thus possible that strain SB10 may produce more antimicrobial compounds than reported here, as suggested by the APE Ec biosynthetic complex. Further research is required to develop these broad-spectrum antimicrobial compounds into drugs that may be used in the fight against microbial infections.

Keywords: Xenorhabdus khoisanae, Antimicrobial complex, Lys-rich PAX lipopeptides, Xenocoumacin, Mass spectrometric analysis

\footnotetext{
* Correspondence: mra@sun.ac.za; LMTD@sun.ac.za

${ }^{2}$ BIOPEP Peptide Group, Department of Biochemistry, Stellenbosch University,

Private Bag X1, Matieland 7602, South Africa

'Department of Microbiology, Stellenbosch University, Private Bag X1,

Matieland 7602, South Africa
}

(c) The Author(s). 2019 Open Access This article is distributed under the terms of the Creative Commons Attribution 4.0 International License (http://creativecommons.org/licenses/by/4.0/), which permits unrestricted use, distribution, and reproduction in any medium, provided you give appropriate credit to the original author(s) and the source, provide a link to the Creative Commons license, and indicate if changes were made. The Creative Commons Public Domain Dedication waiver (http://creativecommons.org/publicdomain/zero/1.0/) applies to the data made available in this article, unless otherwise stated. 


\section{Background}

Xenorhabdus bacteria are in a species-specific association with Steinernema nematodes, i.e. a specific Steinernema sp. is associated with a specific Xenorhabdus sp. [1]. At the beginning of the Xenorhabdus-Steinernema life cycle, nematodes in the infective juvenile phase infect the insect host by entering the mouth, anus or respiratory spiracles [2]. Once inside the insect, the nematodes release the symbiotic bacteria by defecation. Steinernema nematodes produce proteins that suppress the insect's immune response, which allows Xenorhabdus to multiply [3]. The release of exoenzymes and toxins by both mutualists leads to septicaemia and the insect dies within 24 to $48 \mathrm{~h}$ after infection [4-6]. Nematodes reproduce sexually by going through phases $\mathrm{J} 1$ to J4 until resources are depleted, after which they return to the infective juvenile state, acquire symbionts and leave the cadaver in search of a new host.

During the nematode life cycle, Xenorhabdus spp. produce various compounds to create a semi-exclusive environment and prevent colonisation of the host (insect) by other microorganisms [7]. Dutky [8] was the first to suggest that Xenorhabdus produce antimicrobial compounds. However, interest in these antimicrobial compounds only gained momentum 22 years later [9]. Numerous bioactive compounds have since then been detected in the cell-free supernatants of Xenorhabdus spp., including broad-spectrum compounds with activity against bacteria, fungi, insects, nematodes, protists and cancer cells. These compounds range from being small antibiotics, such as xenocoumacins [10], xenortides [11], xenorhabdins [12] and indole derivatives [13], to larger, more complex, compounds such as xenoamicins [14], bicornutin [15] and various antimicrobial peptides (AMPs). Some AMPs are cyclic, such as PAX (peptideantimicrobial-Xenorhabdus) lipopeptides [16], taxlllaids [17], xenobactin [18] and szentiamide [19], while others are linear, such as rhabdopeptides [20], cabanillasin [21] and bacteriocins. Thus far, xenocin and xenorhabdicin, produced by Xenorhabdus nematophila [22, 23], are the only bacteriocins characterised to structural level. Bacteriocins have also been isolated from Xenorhabdus bovienii and Xenorhabdus beddingii, but they have not been fully characterised [24].

Gualtieri and co-workers [16] identified five lysine-rich PAX peptides from $X$. nematophila F1/1 with strong antifungal activity against Fusarium oxysporum and several plant pathogenic fungi. Fuchs et al. [25] identified another 13 lysine-rich PAX peptides produced by $X$. nematophila HGB081. The PAX peptides have an identical backbone structure, with cyclization occurring between the $\varepsilon$-amino group of $\mathrm{Lys}^{3}$ and the C-terminus. According to NMR studies, the fatty acids in PAX peptides from $X$. nematophila F1/1 are iso-branched [26], whilst those of $X$. nematophila HGB081 are straight-chain fatty acids [25]. Three NRPS genes (paxA, $\operatorname{paxB}$ and $\operatorname{pax} C$ ) encode biosynthesis of the PAX peptides [25]. PAX peptides have antifungal and antibacterial activity, but they are not cytotoxic towards insects [25, 26].

The antimicrobial compounds are generally used in combination with the associated nematodes as biological control agents in the agricultural industry [26-30]. A number of antimicrobial compounds produced by Xenorhabdus spp. have been patented [31-37]. For the first time we describe a number of PAX lipopeptides from the A-group and xenocoumacin that are coproduced by Xenorhabdus khoisanae SB10.

\section{Results and discussion}

Xenorhabdus bacteria are known to produce various antimicrobial compounds, but it is a highly neglected antimicrobial source that has not been exploited to its full potential. Although many genes relevant to antimicrobial compound biosynthesis have been identified in Xenorhabdus spp., the isolation, purification, identification and characterisation of antimicrobial compounds have not been done for all species belonging to this genus. Our antiSMASH [38] analysis of the genome of $X$. khoisanae SB10 revealed the presence of the xenocoumacin biosynthetic gene cluster and an APE Ec gene cluster (Fig. 1). The APE Ec gene cluster is widely distributed amongst prokaryotes and is related to secondary metabolites such as aryl polyenes [39]. Identification of the four modules coding for the PAX synthetase complex in the genome of $X$. khoisanae SB10 was accomplished by using tblastx (Table 1). This study, therefore, focussed on the first isolation and confirmation that specific antimicrobial compounds are indeed produced by $X$. khoisanae SB10. The following section describes the purification and characterisation of a selection of antimicrobial compounds from $X$. khoisanae SB10 cultures. A summary of the UPLC-MS data and identification of the compounds in the antimicrobial fractions are presented in Table 2. Detailed analysis utilising ESMS, UPLC-MS and MS ${ }^{\mathrm{e}}$ data on these fractions can be found in the Additional file 1 section.

The first chromatography of the SPC active fractions on $15 \mathrm{RPC}$ resin by FPLC yielded fraction A1 with broad-spectrum antimicrobial activity (Fig. 2a). The SPC A1 fraction was shown to have broad-spectrum activity towards the four target organisms (B. subtilis, E. coli and C. albicans), as well as retaining activity after heating at $121^{\circ} \mathrm{C}$ for $20 \mathrm{~min}$ (results not shown). The latter result indicated marked heat stability of the antimicrobial compounds and eliminated activity related to labile, or volatile compounds and proteins such as proteases. The A1 fraction was further separated with $\mathrm{C}_{18}$-HPLC into seven peaks (Fig. 2b). Fractions B, D and G showed strong UV 


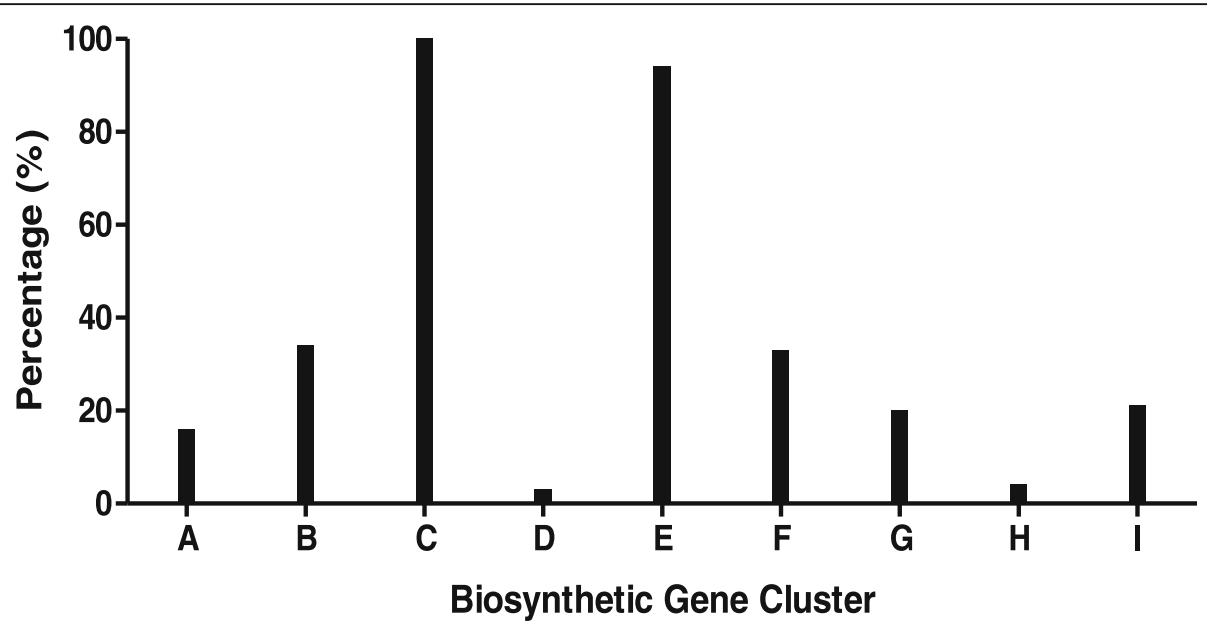

Fig. 1 AntiSMASH results presenting the similarity between the genome of $X$. khoisanae SB10 and biosynthetic gene clusters of Thiomarinol (A), Zeamine (B), Xenocoumacin (C), Lysobactin (D), APE EC (E), Xenoamicin (F), Safracin (G), Taxlllaid (H) and Acinetobactin (I)

absorption (Fig. 2b), suggesting that they did not only contain PAX peptides, but also xenocoumacin and breakdown products. Antimicrobial activity was observed in fractions A to G (Fig. 2b) and is shown in Fig. 3. We first focused on the three major UVabsorbing fractions, B, D and G, as well as C and F (Fig. 2b), for further analysis using high resolution UPLC-MS and UPLC-MS (or MS/MS) analyses (refer to Additional file 1 and Table 2).

The major UPLC-MS peak observed in fraction B contained a small compound with a yellow colour (absorption maximum at $314 \mathrm{~nm}$ ) and a monoisotopic $M_{\mathrm{r}}$ of 406.2104 (Fig. 4b and c, insert shows UV-spectrum). The UPLC-MS ${ }^{\mathrm{e}}$ analysis of the peak, containing its molecular ion with $\mathrm{m} / z 407.2176$ at $4.27 \mathrm{~min}$, yielded six mayor ions (Fig. 5a). The compound was identified as xenocoumacin 2 (expected $m / z=407.2182$ ) based on the characteristic fragmentation pattern. The ion with $\mathrm{m} / \mathrm{z}$ 250.1428 (Fig. 5a) represents the benzopyran-1-one fragment (expected $m / z=250.1443$ ), while the ion with $\mathrm{m} / z$ 158.0788 (Fig. 5a) correlates to the remaining fragment with pyrrolidine as $\mathrm{R}$ group (expected $\mathrm{m} / z=158.0817$ ) [37]. Ions with $\mathrm{m} / \mathrm{z} 176.0699,190.0849,215.1058$ and 232.1324 (Fig. 5b) are the hydration and dehydration products of the two main fragments with $\mathrm{m} / \mathrm{z}$ 158.0788 and 250.1428 (Fig. 5b). This identification of a xenocoumacin produced by $X$. khoisanae SB10, was supported by antiSMASH [39] results of $100 \%$ similarity between the genome of strain SB10 and the xenocoumacin biosynthetic gene cluster (refer to Fig. 1).

Fraction B also contained an earlier eluting peak at $2.90 \mathrm{~min}$ (refer to Fig. 4a) of a larger compound with $\mathrm{m} /$ $z 1052.7948\left(M_{\mathrm{r}}=1051.7870\right)$. The fragmentation pattern of the ion with $\mathrm{m} / z 1052.7948$ showed a neutral loss of 128.09 from the major fragments, which is indicative of the loss of multiple Lys-residues from a peptide chain. The majority of ions with a neutral fragment loss also had resultant dehydration products. This dehydration is the consequence of a fragmentation reaction at the Cterminal of a Lys residue that leads to cyclisation in which the amino group of the Lys side chain participates, similar to fragmentation reactions found for ornithine-containing peptides [40]. Ions with $\mathrm{m} / \mathrm{z}$ 129.1015 and 84.0799 also maps to Lys and its immonium ion. The ion spectrum of $\mathrm{m} / \mathrm{z} 1052.7948$ and proposed fragmentation pattern is presented in Fig. 6. From this spectrum we were able to map the sequence to a Lys-rich lipopeptide from the PAX peptide group. This particular peptide was identified as PAX1' with the R group as (3R)-3-hydroxytetradecanoyl coupled to GlyLys $_{2}$-cyclo $\left(\right.$ Lys $_{4}$ ) with an expected $M_{\mathrm{r}}$ of 1051.7845 (refer to Fig. 6 for structure). Although fraction B did not show

Table 1 Identification of the four modules coding for the PAX synthetase complex in the genome of X. khoisanae SB10

\begin{tabular}{lllllllll}
\hline Gene & $\begin{array}{l}\text { Protein } \\
\text { product }\end{array}$ & $\begin{array}{l}\text { Synthetase } \\
\text { Protein }\end{array}$ & $\begin{array}{l}\text { Presumed } \\
\text { function }\end{array}$ & $\begin{array}{l}\text { Position on } \\
\text { Chromosome }\end{array}$ & Identity (\%) & Positivity (\%) & Origin & $\begin{array}{l}\text { Accession } \\
\text { number }\end{array}$ \\
\hline xcn1_2784 & PaxT & XpsD & ABC-transporter & Node 4-50,691 to 52,328 & $76(416 / 549)$ & $88(487 / 549)$ & X. bovienii SS-2004 & CBJ81280.1 \\
xcn1_2783 & PaxA & XpsA & NRPS & Node 4-52,788 to 56,036 & $62(678 / 1098)$ & $76(836 / 1098)$ & X. bovienii SS-2004 & CBJ81279.1 \\
xcn1_2782 & PaxB & XpsB & NRPS & Node 4-66,118 to 76,893 & $63(2265 / 3614)$ & $76(2747 / 3614)$ & X. bovienii SS-2004 & CBJ81277.1 \\
xcn1_2781 & PaxC & XpsB & NRPS & Node 4-56,079 to 66,107 & $69(2307 / 3356)$ & $81(2733 / 3356)$ & X. bovienii SS-2004 & CBJ81278.1 \\
\hline
\end{tabular}


Table 2 Summary of the antimicrobial compounds in the three main absorbing fractions that were identified using UPLC-MS and UPLC-MS ${ }^{e}$. PAX peptide identities and names are from Fuchs et al. [32]

\begin{tabular}{|c|c|c|c|c|c|c|}
\hline Fraction & UPLC Rt (min) & $m / z$ of major $[\mathrm{M}+\mathrm{H}]^{+}$ & Compound $M_{r}^{a}$ & Theoretical $M_{\mathrm{r}}^{\mathrm{b}}$ & Mass error $(\mathrm{ppm})^{c}$ & Proposed compound identity \\
\hline \multirow[t]{2}{*}{ B } & 4.27 & 407.2182 & 406.2104 & 406.2104 & 0.0 & Xenocoumacin $2\left(\mathrm{C}_{21} \mathrm{H}_{30} \mathrm{~N}_{2} \mathrm{O}_{6}\right)$ \\
\hline & 2.93 & 1052.7948 & 1051.7870 & 1051.7845 & 2.4 & $\mathrm{PAX}^{\prime}\left(\mathrm{C}_{14} \mathrm{H}_{27} \mathrm{O}_{2}\right) \mathrm{GK}_{6}$ \\
\hline \multirow[t]{2}{*}{ C } & 3.18 & 1078.8119 & 1077.8041 & 1077.8001 & 3.7 & PAX7E1* $\left(\mathrm{C}_{16} \mathrm{H}_{29} \mathrm{O}_{2}\right) \mathrm{GK}_{6}$ \\
\hline & 3.42 & 1070.8091 & 1069.7921 & 1069.7951 & -2.8 & PAX1L* $\left(\mathrm{C}_{14} \mathrm{H}_{29} \mathrm{O}_{3}\right) \mathrm{GK}_{6}$ \\
\hline D & 3.29 & 1066.8097 & 1065.7965 & 1065.8001 & -3.4 & $\mathrm{PAX3}^{\prime}\left(\mathrm{C}_{15} \mathrm{H}_{29} \mathrm{O}_{2}\right) \mathrm{GK}_{6}$ \\
\hline \multirow[t]{4}{*}{$\mathrm{F}$} & 3.47 & 1054.8115 & 1053.8012 & 1053.8001 & 1.0 & $\mathrm{PAX} 1 \mathrm{~L}-\mathrm{DH} \mathrm{H}^{*}\left(\mathrm{C}_{14} \mathrm{H}_{29} \mathrm{O}_{2}\right) \mathrm{GK}_{6}$ \\
\hline & $3.32,3.47^{\#}$ & 1080.8286 & 1079.8191 & 1079.8158 & 3.1 & $\operatorname{PAX7}\left(\mathrm{C}_{16} \mathrm{H}_{31} \mathrm{O}_{2}\right) \mathrm{GK}_{6}$ \\
\hline & 3.71 & 1078.8096 & 1077.8002 & 1077.8001 & 0.1 & PAX7E2* $\left(\mathrm{C}_{16} \mathrm{H}_{29} \mathrm{O}_{2}\right) \mathrm{GK}_{6}$ \\
\hline & 3.88 & 1078.8070 & 1077.7979 & 1077.8001 & -2.0 & PAX7E3* $\left(\mathrm{C}_{16} \mathrm{H}_{29} \mathrm{O}_{2}\right) \mathrm{GK}_{6}$ \\
\hline \multirow[t]{4}{*}{ G } & 2.51 & 1050.7723 & 1049.7623 & 1049.7688 & -6.2 & PAX5 $\left(\mathrm{C}_{14} \mathrm{H}_{25} \mathrm{O}_{2}\right) \mathrm{GK}_{6}$ \\
\hline & 2.90 & 1052.7936 & 1051.7821 & 1051.7845 & 2.3 & $\mathrm{PAX1}^{\prime}\left(\mathrm{C}_{14} \mathrm{H}_{27} \mathrm{O}_{2}\right) \mathrm{GK}_{6}$ \\
\hline & 3.57 & 1080.8280 & 1079.8161 & 1079.8158 & 0.3 & PAX7 $\left(\mathrm{C}_{16} \mathrm{H}_{31} \mathrm{O}_{2}\right) \mathrm{GK}_{6}$ \\
\hline & 3.83 & 1106.8412 & 1105.8294 & 1105.8314 & -1.8 & PAX8 $\left(\mathrm{C}_{18} \mathrm{H}_{33} \mathrm{O}_{2}\right) \mathrm{GK}_{6}$ \\
\hline
\end{tabular}

${ }^{a}$ Experimental monoisotopic $M_{\mathrm{r}}$ of compound was calculated using the TOF transform or MaxEnt3 function in the MassLynx 4.01 software package

${ }^{\mathrm{b}}$ Theoretical monoisotopic $M_{\mathrm{r}}$ of compound was calculated from accurate monoisotopic $M_{\mathrm{r}}$ of Lys $=128.09496$ and Gly $=57.02146$, and monoisotopic $A_{\mathrm{r}}$ of $\mathrm{O}=15.9949146 ; \mathrm{H}=1.0078250, \mathrm{~N}=14.0030740$ and $\mathrm{C}=12.0000000$

${ }^{c}$ Mass error in parts per million $(\mathrm{ppm})=10^{6} \times\left\{M_{\mathrm{r}}\right.$ (theoretical) $-M_{\mathrm{r}}$ (experimental) $\} / M_{\mathrm{r}}$ (theoretical)

"Early elution of broad peak, fronting and tailing due to aggregation at high concentration

*Putative identification as PAX peptides from peptide moiety fragments and accurate mass determination, $\mathrm{E}$ denotes an enoyl group, $\mathrm{L}$ denotes linear, $\mathrm{DH}$ denotes dehydroxylated, structure of R-group was not elucidated

Refer to Additional file 1 for UPLC-MS, ESMS and MS/MS data on all the compounds

high activity against $E$. coli Xen 14, high activity was recorded against all other target strains when purified PAX1' and xenocoumacin were combined in a 1:1 ratio (Fig. 3). This result warrants further investigation to determine if the activity between PAX peptides and small water soluble xenocoumacins are synergistic.

With the identification of the PAX1' peptide there was a high probability of other PAX peptides in the HPLC fractions. In fraction $\mathrm{C}$ two more PAX peptides were found, namely a PAX peptide $(\mathrm{m} / z$ 1070.8091) which correlated to a linear analogue of PAX1' (denoted PAX1'L) and a novel PAX peptide from the A group at $m / z 1078.8119$, that correlated to a PAX7 with an enoyl group in the lipid chain, denoted PAX7E1. This putative identification was done from the accurate $M_{\mathfrak{v}}$ fragmentation pattern of the peptide moiety (refer to Additional file 1) and the fact that it eluted just before PAX7. This elution pattern correlated very well with that of PAX1' and its 7-enoyl analogue PAX5 (see discussion below and Table 2). As there is already this identified 7-enoyl analogue in the PAX group, it is possible PAX7E1 is also a 7-enoyl analogue. We were, however, not able to confirm the structure of the $\mathrm{R}$ group with our in-analysis $\mathrm{MS}^{\mathrm{e}}$ methodology, as the CID energy only released the lipid moiety and limited fragmentation was achieved. Fraction D contained PAX3' $(\mathrm{m} / z$ 1066.8097) and fraction F contained PAX7 $(\mathrm{m} / z$ 1080.8286). This fraction also contained UV absorbing compounds (refer to Fig. 2) that are possibly the result of xenocoumacin breakdown (detected compounds with $m / z$ 250.2162, 268.2263, $270.2425,286.2369)$. The high PAX7 concentration in fraction F led to UPLC peak broadening, possibly due to the aggregation of this lipopeptide. Although PAX7 was the major compound in fraction $\mathrm{F}$, this fraction also contained other PAX-like peptides. Two low abundance peptides with different elution times, but with the same $m / z$ as PAX7E1 with a putative double bond (enoyl) in lipid chain, were observed at 3.71 and $3.88 \mathrm{~min}(\mathrm{~m} / \mathrm{z}$ 1078.8096 and $m / z$ 1078.8096, denoted PAX7E2 and PAX7E3). PAX7E2 displayed similar peptide chain fragments to that of PAX7 and PAX7E1, suggesting that the difference in elution time may or due to the position of the putative double bound in the lipid chain (refer to Additional file 1). PAX7E3 co-eluted with various compounds, so the fragmentation pattern was inconclusive although many similar Lys derived fragments were observed. Alternatively, it could be that PAX7E2 and PAX7E3 elutes later than PAX7E1 due to aggregation with PAX7 and other compounds, rather than structural differences. A peptide with $\mathrm{m} / z 1054.8090$ co-eluted with the PAX7 peptide. From the accurate $M_{\mathrm{r}}$ it was derived that this peptide could be a linear PAX1' without a hydroxyl-group (denoted PAX1'L-DH), but the structure remains unconfirmed due to the co-elution. Fraction G contained a number of PAX peptides, namely PAX5 $(\mathrm{m} /$ $z$ 1050.7723), PAX1' $(\mathrm{m} / z$ 1052.7936), PAX7 $(\mathrm{m} / z$ $1080.8280)$ and PAX8 $(\mathrm{m} / z$ 1106.8412). The fact that the same PAX peptides eluted in more than one fraction is 


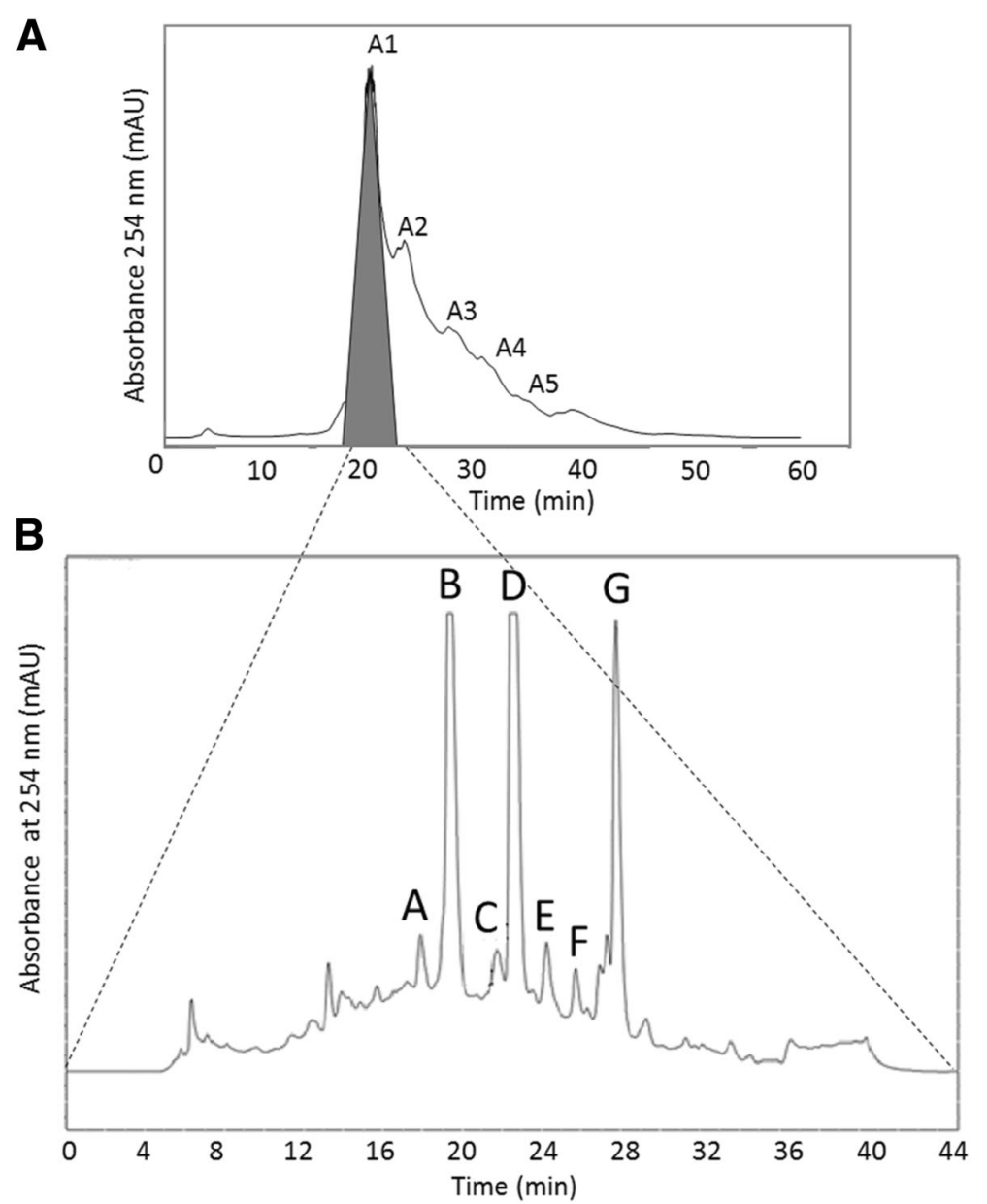

Fig. 2 Representative chromatograms depicting the isolation of antimicrobial fractions from the $X$. khoisanae SB10 culture extracts. a Separation of SPC active fractions on 15 RPC resin by FPLC, with a linear gradient of 10 to $55 \%(\mathrm{v} / \mathrm{v})$ acetonitrile containing $0.1 \%(\mathrm{~V} / \mathrm{V})$ TFA. b $\mathrm{C}_{18}$ HPLC chromatography of the fraction A1 in graph A (FPLC active fraction). A linear gradient from 25 to $45 \%$ acetonitrile containing $0.1 \%$ TFA was used. The peak fractions denoted A-G in graph B displayed antimicrobial activity

possibly due to the formation of hetero-oligomers by the different lipopeptides, leading to elution at different acetonitrile concentrations during reverse phase chromatography. As in fraction $\mathrm{D}$, fraction $\mathrm{G}$ also contained some UV absorbing compounds (refer to Fig. 2) that are possibly the result of xenocoumacin breakdown. Examples of the UPLC-MS chromatograms and spectra of the five most abundant PAX peptides are presented in Fig. 7. The primary structures of the known PAX peptides that were found in this study are given in Fig. 8. We were able to confirm the peptide sequence of most of the identified PAX peptides with our UPLC-MS $S^{\mathrm{e}}$ procedure, except those that were found in very low concentrations. Similar fragment patterns to that depicted in Fig. 5 for PAX1' were observed for the PAX peptides discussed above (refer to Additional file 1). This discovery and identification of the PAX lipopeptides were supported by the identification of the four modules coding for the PAX synthetase complex in the genome of $X$. khoisanae by our tblastx study (refer to Table 1).

\section{Conclusions}

It was not surprising to discover the production of various antimicrobial compounds by $X$. khoisanae due to the Xenorhabdus-Steinernema-insect host tripartite interaction [1]. The known PAX peptides have only been isolated from $X$. nematophila, however, this is the first report of PAX peptides produced by X. khoisanae. PAX lipopeptides were first characterised by Gaulteri et al. [16] and to date 13 unique PAX peptides have been reported [34]. Our UPLC-MS and $\mathrm{MS}^{\mathrm{e}}$ analyses and identification of PAX peptides and compounds in the antimicrobial complex of $X$. khoisanae SB10 have not been exhaustive, because of the complexity of many fractions containing labile compounds or unresolved compounds. There are certainly more antimicrobial compounds to discover in this strain's natural antimicrobial complex in 


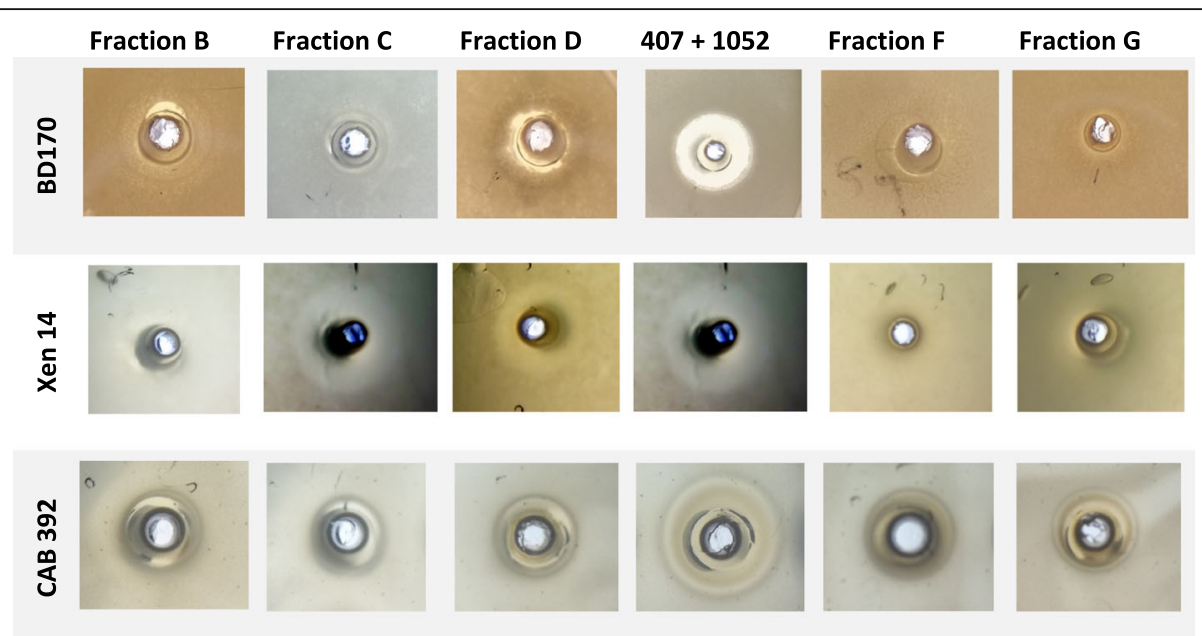

Fig. 3 Antimicrobial activity of the factions collected (fractions B, C, D, F and $\mathrm{G}$ ) and the combined activity of purified xenocoumacin $2(\mathrm{~m} / \mathrm{z} 407)$ and $\operatorname{PAX}^{\prime} 1^{\prime} 1(\mathrm{~m} / \mathrm{z}$ 1052) from the compound library of the Department of Microbiology. Growth inhibition is observed as zones surrounding the wells. Activity BD170 = Bacillus subtilis subsp. subtilis, Xen 14=Escherichia coli and CAB $392=$ Candida albicans

future studies, such as other xenocoumacins, xenorhabdins and compounds from the aryl polyene group, as indicated by the detection of the APE Ec biosynthetic complex (refer to Fig. 1). Because we consistently found the PAX lipopeptides and xenocoumacin in all the $X$. khoisanae SB10 culture extracts, we focused in this study on these compounds. Possible metabolites and degradation products related to the xenocoumacins were observed in some fractions (D and G), but after in-depth analysis of all the chromatographic fractions using UPLC-MS only one intact xenocoumacin, namely xenocoumacin 2 , was identified in fraction $B$. It is possible that other xenocoumacins were also produced, but that the more labile compounds were lost during purification in a highly acidic environment. We were, however, able to identify five known Lys-rich lipopeptides from the PAX A-group with PAX1' and PAX7 being the most abundant. We also discovered two putative linear PAX analogues, which could possibly be metabolic precursors of PAX1'. PAX7 could be related to the three unknown PAX peptides with the same $M_{\mathrm{r}}$ of 1077.80 but different retention on a reverse phase matrix. From their $M_{\mathrm{v}}$ fragmentation patterns and UPLC elution behaviour we putatively classified the three peptides as enoyl-derivatives of PAX7. We were, however, unable to confirm the R-group because of low abundance and co-elution, as well as limited fragmentation of the R-group in $\mathrm{MS}^{\mathrm{e}}$ mode.

This study is the first to identify both the PAX peptides and a xenocoumacin in the antimicrobial complex of a Xenorhabdus species, as well as the first study on the antimicrobial complex of the Southern African $X$. khoisanae SB10. This report also highlights the natural tendency of Xenorhabdus species to produce antimicrobial complexes consisting of small antibiotics and AMPs. With the rising antibiotic resistance it may be wise to consider combining AMPs and small antibiotics, mimicking Xenorhabdus-type antimicrobial complexes.

\section{Methods}

Bacterial strains, growth media and growth conditions Xenorhabdus khoisanae SB10 was maintained on NBTA [41], consisting of nutrient agar supplemented with bromothymol blue $(0.025 \%, w / v)$ and TTC $(0.004 \%, w / v)$. Incubation was at $30^{\circ} \mathrm{C}$. Bacillus subtilis subsp. subtilis BD170, Escherichia coli Xen 14 and Candida albicans CAB 392 were used as targets in the testing for antimicrobial activity. The bacteria and yeast were incubated at $37^{\circ} \mathrm{C}$. Bacteria were cultured on Brain Heart Infusion agar (Biolab Diagnostics, Gauteng, South Africa) and $C$. albicans on Potato Dextrose Agar (PDA, Biolab Diagnostics).

\section{Isolation of antimicrobial compounds}

XAD-16 beads were activated by treating with $80 \%$ isopropanol containing $0.1 \%(v / v)$ TFA and added to TSB. After $30 \mathrm{~min}$ at $4{ }^{\circ} \mathrm{C}$ on an orbital shaker $(100 \mathrm{rpm})$, the XAD-16 beads were removed and the medium autoclaved. $X$. khoisanae SB10 was inoculated into $5 \mathrm{ml}$ untreated TSB and incubated at $26^{\circ} \mathrm{C}$ for $24 \mathrm{~h}$ on a rotating wheel. The culture was added to $5 \mathrm{~g}$ activated XAD-16 beads, spread-plated onto XAD-16-treated TSB agar in petri dishes with a diameter of $135 \mathrm{~mm}$ and incubated at $26^{\circ} \mathrm{C}$ for $96 \mathrm{~h}$. Beads were collected from the plates and washed with sterile deionised water to remove the cells. Water was removed from beads by vacuum suction. The beads were washed with $150 \mathrm{~mL} \mathrm{30 \% (v/v)}$ ethanol for $15 \mathrm{~min}$ at $4{ }^{\circ} \mathrm{C}$ on an orbital shaker (100 rpm) . Ethanol was removed by vacuum suction and the beads were washed with sterile deionised water. Amphipathic 


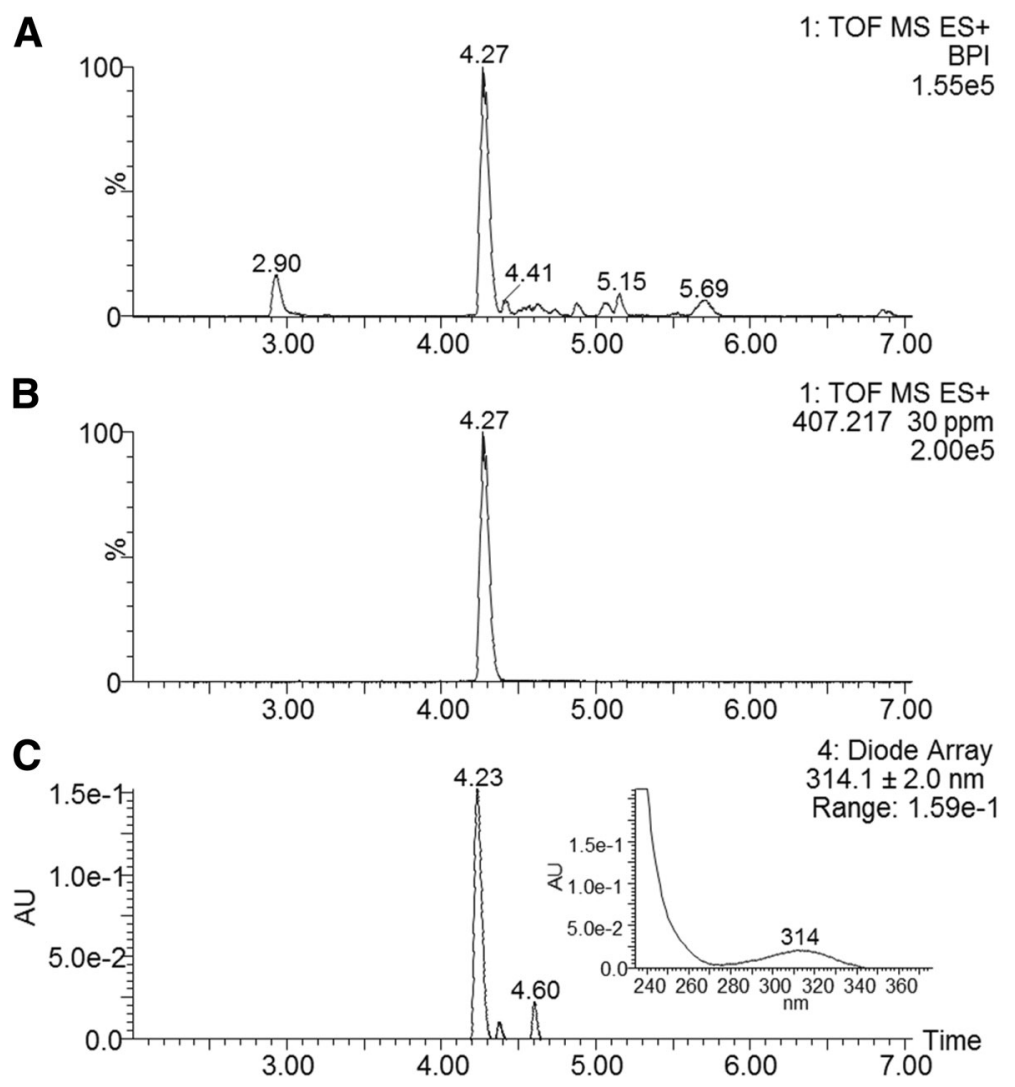

Fig. 4 The UPLC profiles of fraction B collected from $C_{18}-H P L C$ (refer to Fig. 2 b). The top chromatogram (a) shows the base peak intensity mass chromatogram of fraction B and the middle chromatogram (b) the mass extracted chromatogram for the molecular ion with $\mathrm{m} / \mathrm{z} 407.217$ at 30 ppm tolerance. The bottom chromatogram (c) shows the spectrophotometric profile at $314 \mathrm{~nm}$ and the insert shows the UV spectrum of the peak at $4.23 \mathrm{~min}$

compounds were liberated from the beads, using $70 \%(v / v)$ isopropanol containing $0.1 \%(v / v)$ TFA (isopropanol-TFA). The eluent was filtered through a $0.45 \mu \mathrm{M}$ cellulose nitrate filter and the isopropanol removed by using a rotary evaporator (RotaVapor ${ }^{\circ} \mathrm{R}$ 114, Büchi).

\section{Purification of antimicrobial compounds}

The concentrated eluent was subjected to reverse phase chromatography on a $10 \mathrm{ml}$ Sep-Pak C18 column (Waters, Milford, USA) on Perista Pump SJ-1211 (Chromatograph ATTO corporations, Tokyo, Japan). The column was washed with deionised water and compounds eluted by using a stepwise gradient ranging from 10 to $70 \%(v / v)$ isopropanol in analytical quality water with constant $0.1 \%$ TFA $(v / v)$ in solvent system. The gradient was created with $10 \%$ increments per $10 \mathrm{~min}$ at $2 \mathrm{~mL} /$ minute flowrate. The fractions (denoted SPC fractions) were dried by rotary evaporation and tested for antimicrobial activity using the agar-well diffusion assay as described elsewhere.

Active SPC fractions were loaded onto a HiScale column $(100 \times 16 \mathrm{~mm})$ packed with $15 \mathrm{RPC}$ resin (GE
Healthcare, South Africa) fitted to fast protein liquid chromatography (FPLC, ÄKTA purifier, GE Healthcare, South Africa). Fractions were eluted by a linear gradient of 10 to $55 \%$ B over $30 \mathrm{~min}$, at a flow rate of $2.5 \mathrm{ml} / \mathrm{min}$ (A: analytical quality water containing $0.1 \%, v / v, T F A$; B: HPLC grade acetonitrile containing $0.1 \%, v / v$, TFA). Readings were recorded at $254 \mathrm{~nm}$. Fractions were tested for antibacterial activity against $B$. subtilis subsp. subtilis $\mathrm{BD} 170$, using the agar-well diffusion assay as described elsewhere.

Fractions with antimicrobial activity collected from the ÄKTA purifier (FPLC active fractions) were lyophilized, resuspended in 50\% $(v / v)$ acetonitrile, loaded onto a Discovery BIO Wide Pore $\mathrm{C}_{18}$ HPLC column $(10 \mu \mathrm{m}, \quad 250 \times 10 \mathrm{~mm} ; \quad$ Sigma-Aldrich $)$ and eluted by using a linear gradient (25 to $45 \%$ ) of eluent B over $28 \mathrm{~min}$ at a flow rate of $2.0 \mathrm{ml} / \mathrm{min}$. Further separation was on a Surveyor plus HPLC (Thermo Fisher Scientific, Waltham, Massachusetts, USA). Readings were recorded at $254 \mathrm{~nm}$. Peak fractions were collected, dried and the antimicrobial activity tested as described elsewhere. 

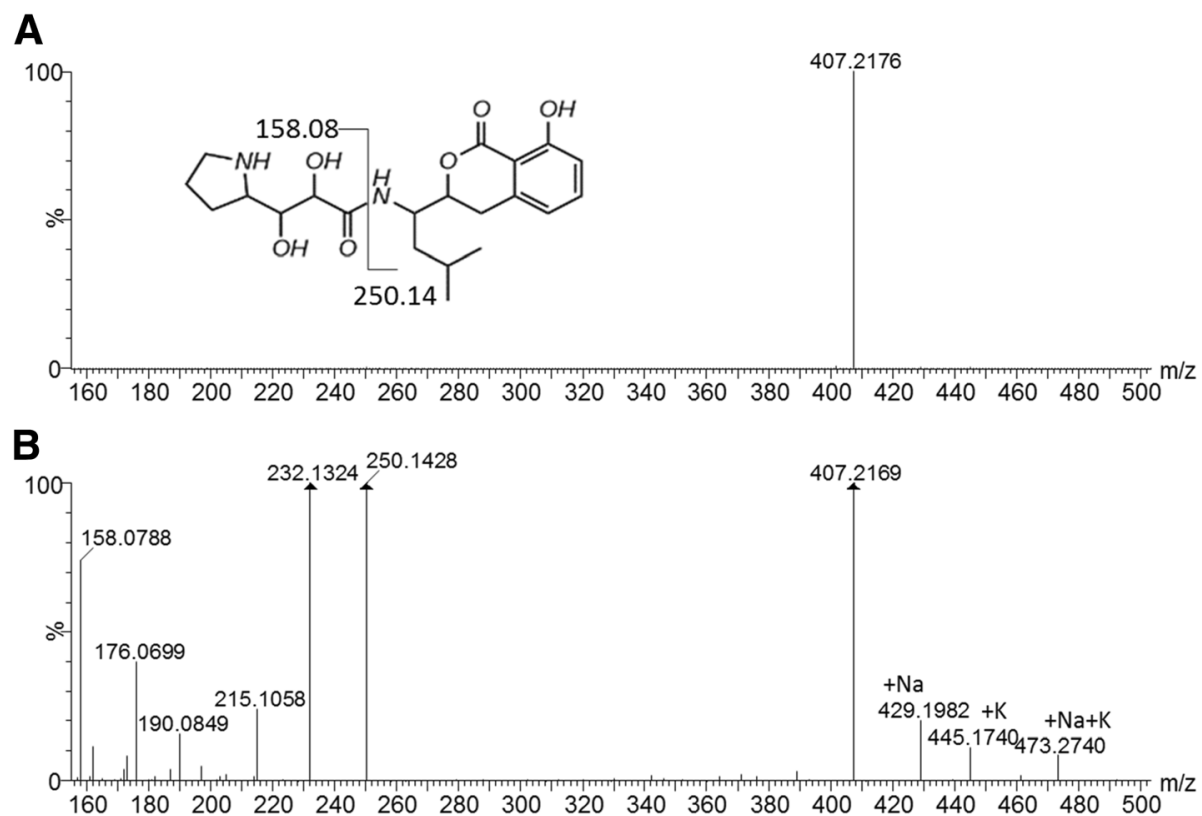

Fig. 5 ESI-MS and CID spectra (generated via MS type analysis) of the main component at 4.23 in fraction B, namely xenocoumacin 2. The component mass spectrum of xenocoumacin 2 (structure insert) is shown in the top spectrum (a) and fragmentation product ion spectrum is shown in the bottom spectrum (b). The two main fragments are indicated on the xenocoumacin 2 structure. Refer to the text for the discussion of the fragmentation of xenocoumacin 2

\section{5}
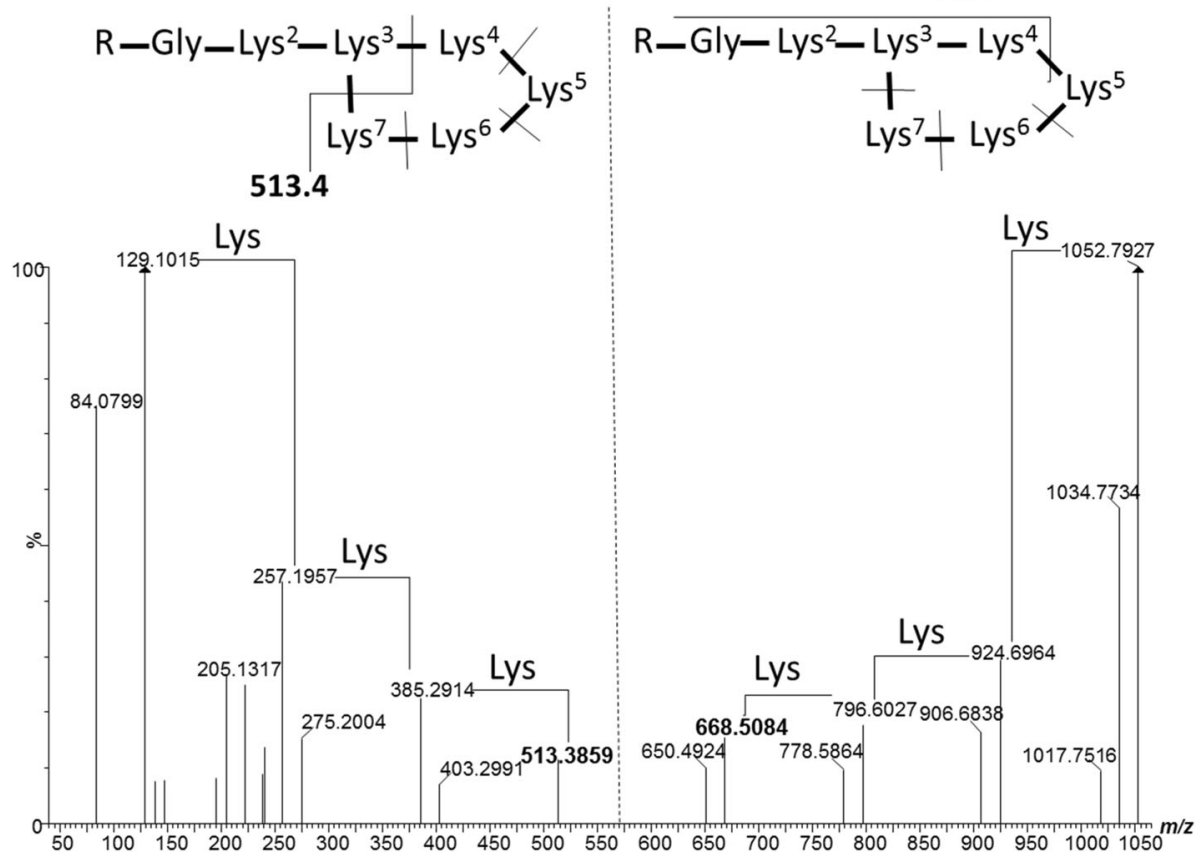

Fig. 6 Representative CID spectrum over $m / z$ 50-1050 of PAX $1^{\prime}$ with intact molecular ion at $m / z=1052.79$. CID analyses were performed over a CE gradient from 20 to $60 \mathrm{eV}$ at a CV of $15 \mathrm{~V}$. The two structures above the spectrum show the fragments that would lead to the 513.4 and 668.5 product ions and their subsequent fragmentation. The R group for PAX $1^{\prime}$ is (3R)-3-hydroxy tetradecanoyl and the side-chain of Lys ${ }^{3}$ links up to the carboxyl group of Lys $^{7}$ to form the ring structure 

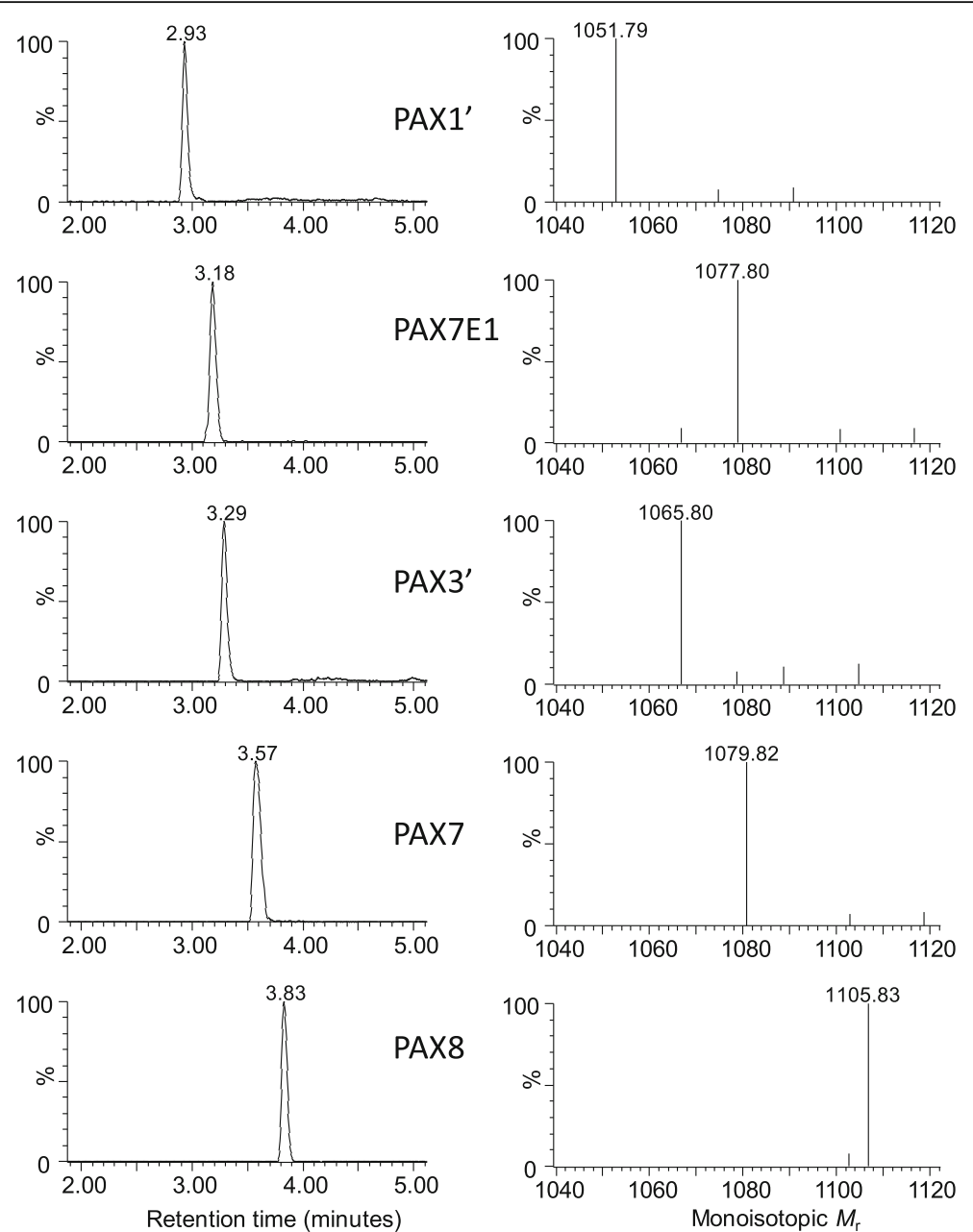

Fig. 7 UPLC-MS profiles (left panel) and ESI-MS spectra (right panel) of the five major PAX lipopeptides that were detected in the antimicrobial fractions of the $X$. khoisanae SB10 culture extracts

\section{Analysis of fractions with ultra-performance liquid chromatography and electrospray ionization mass spectrometry}

Fractions with antimicrobial activity collected from the HPLC were subjected to UPLC, using an Aquity UPLC ${ }^{\text {тм }}$ linked to a Waters Synapt G2 Mass Spectrometer (Waters Corporation, Miliford, USA). This was denoted UPLC-MS. Samples were prepared in 50\% acetonitrile in water $(1: 1, v / \nu)$ at a concentration $200-1000 \mu \mathrm{g} / \mathrm{mL}$. Samples were injected at $1-5 \mu$ via Waters Acquity $\mathrm{UPLC}^{\mathrm{TM}}$ and chromatography was monitored in positive ESI mode and via PDA (230-400 nm). Separation of the components in each HPLC fraction were done on an Acquity UPLC ${ }^{\oplus}$ HSS T3 $\mathrm{C}_{18}$ column $(1.8 \mu \mathrm{m}$ particle size, $2.1 \times 150 \mathrm{~mm}$, Waters Corporation, Dublin, Ireland) . Chromatography was done with analytical quality water modified with $0.1 \%(v / v)$ formic acid as eluent A and acetonitrile modified with $0.1 \%(v / v)$ formic acid as eluent B. The gradient developed at flow rate of $300 \mu \mathrm{l} /$ min was as follows: 0 to $0.5 \mathrm{~min}$ at $40 \% \mathrm{~B}$, linear gradient from 40 to $95 \%$ B from 0.5 to $11 \mathrm{~min}$ and 11 to 14 min at $95 \% \mathrm{~B}$. The rest of the instrument settings for the UPLC-MS mode were as follows: cone voltage set at 15 $\mathrm{V}$, a capillary voltage of $2.5 \mathrm{kV}$, cone voltage of $15 \mathrm{~V}$, extraction cone voltage $4 \mathrm{~V}$, source temperature of $120^{\circ} \mathrm{C}$, desolvation gas of $650 \mathrm{l} / \mathrm{h}$ and desolvation temperature of $275^{\circ} \mathrm{C}$. Data were collected in positive mode by scanning through $m / z=100$ to 2000 in centroid mode at a rate of $0.2 \mathrm{scans} / \mathrm{sec}$.

High resolution collisionally induced dissociation (CID) analyses were done in the $\mathrm{MS}^{\mathrm{e}}$ mode (tandem MS or MS/MS) during the UPLC-MS and monitored on a second MS channel. CID were done at a collision energy gradient of 20 to $60 \mathrm{eV}$ at $1 \mathrm{~s} \mathrm{MS/MS} \mathrm{scan} \mathrm{time.} \mathrm{Data}$ were collected in the second mass analyser (MS2) through $\mathrm{m} / z=40$ to 1500 in centroid mode. The rest of the instrument settings were as described above. To ensure reliable high-resolution MS data, the MS instruments 


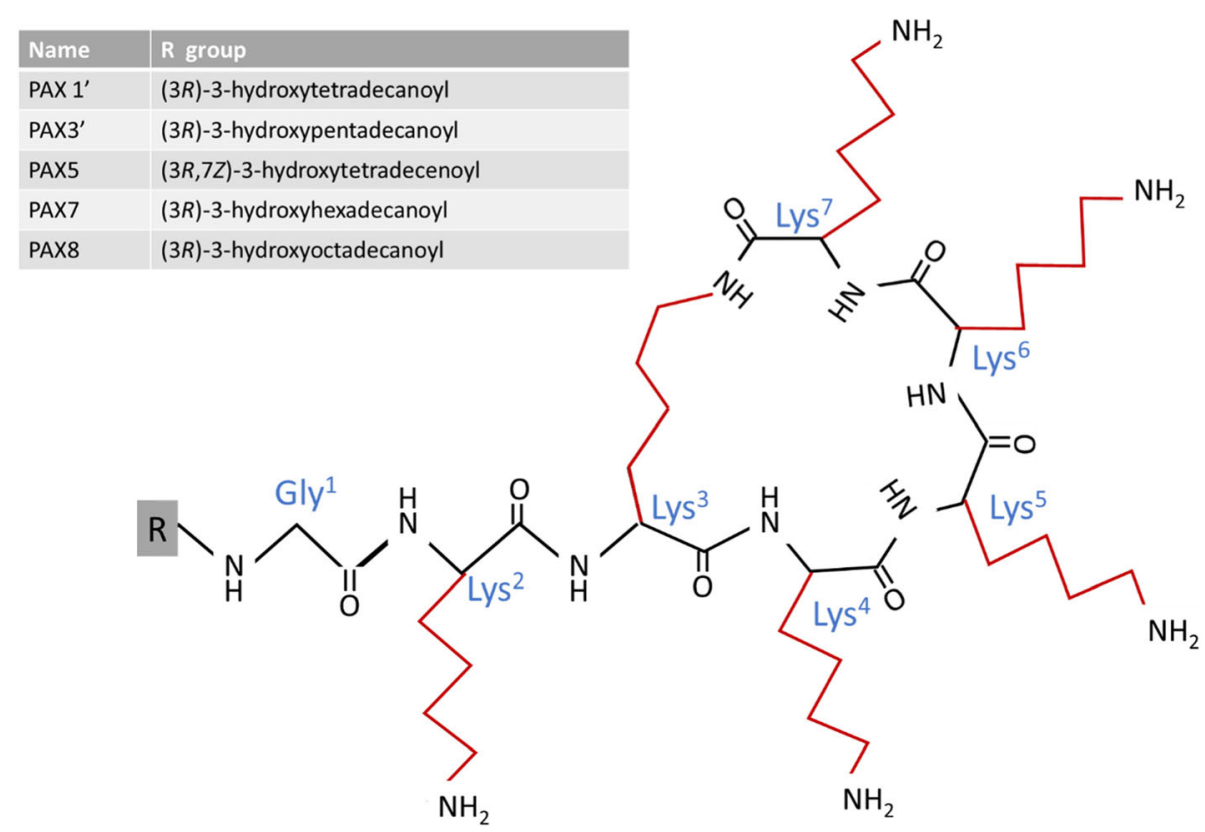

Fig. 8 Primary structure of five known PAX lipopeptides [32] that were detected in the antimicrobial fractions of the X. khoisanae SB10 culture extracts

were calibrated with sodium formate. Single point lock spray using leucine encephalin $(m / z=556.2771)$ as calibrant was used during analysis to compensate for any $m / z$ drift.

\section{Antimicrobial activity of fractions from purification}

SPC active fractions were suspended in analytical quality water, containing $0.1 \%(v / v)$ TFA to $350 \mathrm{mg} / \mathrm{ml}$. The antimicrobial activity of fractions was tested using an agar-well diffusion assay in a micro well titre plate. In short, the appropriate growth media containing 1.0\% ( $w /$ v) agar was seeded with a dense 12-h-old culture of $B$. subtilis subsp. subtilis BD170, E. coli Xen $14(1.0 \%, v / v)$ or C. albicans CAB $392(1.0 \%, v / v)$. Wells were made into the agar and $15 \mu \mathrm{L}$ of each fraction dispensed into a well. Plates were incubated for 24 to $48 \mathrm{~h}$ at $37^{\circ} \mathrm{C}$. A clear zone surrounding the well indicated activity. Analytical quality water, containing $0.1 \%(\mathrm{v} / \mathrm{v})$ TFA, was used as negative control. Ciprofloxacin was used as positive control for B. subtilis subsp. subtilis BD170 and E. coli Xen 14 and amphotericin B for C. albicans CAB 392.

\section{Temperature stability}

SPC active fractions of $350 \mathrm{mg} / \mathrm{mL}$ were prepared in MilliQ water, containing $0.1 \%(\mathrm{v} / \mathrm{v})$ TFA. The suspension was autoclaved for $20 \mathrm{~min}$ and tested for antimicrobial activity against $B$. subtilis subsp. subtilis BD170, using the agar-well diffusion assay as described elsewhere. Plates were incubated at $37^{\circ} \mathrm{C}$ for $24 \mathrm{~h}$. The diameter of growth inhibition zones was recorded and compared to controls. This was done by using the software program ImageJ (v. 1.48).

\section{Additional file}

Additional file 1: Detailed mass spectrometric analysis of the chromatographic fractions of $X$. khoisanae extracts. (PDF 471 kb)

\section{Abbreviations}

FPLC: Fast protein liquid chromatography; HPLC: High pressure liquid chromatography; MS: Mass spectrometer/mass spectrometry; NRPS: Nonribosomal peptide synthetase; PAX: Peptide-antimicrobial-Xenorhabdus; TFA: Trifluoroacetic acid; TSB: Tryptic soy broth; TTC: Triphenyl tetrazolium chloride; UPLC: Ultraperformance liquid chromatography

\section{Acknowledgements}

The authors wish to thank the staff of the LCMS Central Analytical facility at Stellenbosch University for their invaluable assistance in mass spectrometric analyses.

\section{Authors' contributions}

JD conducted the experiments; MR analysed the data; EB assisted in data analyses; ADVS, SMD and LMTD supervised the research. All authors contributed to the writing of the paper, and read and approved the final manuscript.

\section{Funding}

The project was funded by a South African National Research Foundation grant to LMTD with contributions by MR from the BIOPEP Peptide Fund.

\section{Availability of data and materials}

The datasets generated and/or analysed during the current study are not publicly available due to the preparation of a patent, but are available from the corresponding author (LMTD) on reasonable request.

Ethics approval and consent to participate

Not applicable.

\section{Consent for publication}

Not applicable.

\section{Competing interests}

The authors declare that they have no competing interests. 
Received: 8 August 2018 Accepted: 31 May 2019

Published online: 13 June 2019

\section{References}

1. Thomas GM, Poinar GO. Xenorhabdus gen. Nov., a genus of entomopathogenic, nematophilic bacteria of the family Enterobacteriaceae. Int J Syst Bacteriol. 1979; 29:352-60

2. Poinar GO. Biology and taxonomy of Steinernematidae and Heterorhabtididae. In: Gaugler R, Kaya HK, editors. Entomopathogenic nematodes in biological control. Boca Raton: USA: CRC Press; 1990. p. 365.

3. Gotz P, Boman A, Boman HG. Interactions between insect immunity and an insect-pathogenic nematode with symbiotic bacteria. Proc R Soc B Biol Sci. 1981;212:333-50

4. Dunphy GB, Webster JM. Antihemocytic surface components of Xenorhabdus nematophilus var. dutki and their modification by serum nonimmune larvae of Galleria mellonella. J Invertebr Pathol. 1991;58:40-51.

5. Yang J, Zeng H-M, Lin H-F, Yang X-F, Liu Z, Guo L-H, Yuan J-J, Qiu D-W. An insecticidal protein from Xenorhabdus budapestensis that results in prophenoloxidase activation in the wax moth, Galleria mellonella. J Invertebr Pathol. 2012;110:60-7.

6. Burman M. Neoaplectana carpocapsae: toxin production by axenic insect parasitic nematodes. Nematologica. 1982;28:62-70.

7. Webster JM, Chen G, Hu K, Li J. Bacterial metabolites. In: Gaugler R, editor. Entomopathogenic nematology. New York: CAB International; 2002. p. 99-114.

8. Dutky SR. Insect microbiology. Adv Appl Microbiol. 1959;1:175-200.

9. Paul VJ, Frautschy S, Fenical W, Nealson KH. Antibiotics in microbial ecology. J Chem Ecol. 1981;7:589-97.

10. Mclnerney BV, Taylor WC, Lacey MJ, Akhurst RJ, Gregson RP. Biologically active metabolites from Xenorhabdus spp., part 2. Benzopyran-1-one derivatives with gastroprotective activity. J Nat Prod. 1991;54:785-95.

11. Lang G, Kalvelage T, Peters A, Wiese J, Imhoff JF. Linear and cyclic peptides from the entomopathogenic bacterium Xenorhabdus nematophilus. J Nat Prod. 2008;71:1074-7.

12. Mclnerney BV, Gregson RP, Lacey MJ, Akhurst RJ, Lyons GR, Rhodes SH, Smith DRJ, Engelhardt LM, White AH. Biologically active metabolites from Xenorhabdus spp., part 1. Dithiolopyrrolone derivatives with antibiotic activity. J Nat Prod. 1991;54:774-84

13. Sundar L, Chang FN. Antimicrobial activity and biosynthesis of indole antibiotics produced by Xenorhabdus nematophilus. J Gen Microbiol. 1993;139:3139-48.

14. Zhou Q, Grundmann F, Kaiser M, Schiell M, Gaudriault S, Batzer A, Kurz M, Bode HB. Structure and biosynthesis of xenoamicins from entomopathogenic Xenorhabdus. Chem - A Eur J. 2013:19:16772-9.

15. Böszörményi E, Érsek T, Fodor AM, Fodor AM, Földes LS, Hevesi M, Hogan JS, Katona Z, Klein MG, Kormány A, Pekár S, Szentirmai A, Sztaricskai F, Taylor RAJ. Isolation and activity of Xenorhabdus antimicrobial compounds against the plant pathogens Erwinia amylovora and Phytophthora nicotianae. J Appl Microbiol. 2009;107:746-59.

16. Gualtieri M, Aumelas A, Thaler J-O. Identification of a new antimicrobial lysine-rich cyclolipopeptide family from Xenorhabdus nematophila. J Antibiot (Tokyo). 2009:62:295-302

17. Kronenwerth M, Bozhüyük KAJ, Kahnt AS, Steinhilber D, Gaudriault S, Kaiser M, Bode HB. Characterisation of taxlllaids A-G; natural products from Xenorhabdus indica. Chem - A Eur J. 2014;20:17478-87.

18. Grundmann F, Kaiser M, Kurz M, Schiell M, Batzer A, Bode HB. Structure determination of the bioactive depsipeptide xenobactin from Xenorhabdus sp. PB30.3. RSC Adv. 2013;3:22072-7.

19. Nollmann Fl, Dowling A, Kaiser M, Deckmann K, Grösch S, Ffrench-Constant R, Bode HB. Synthesis of szentiamide, a depsipeptide from entomopathogenic Xenorhabdus szentirmaii with activity against Plasmodium falciparum. Beilstein J Org Chem. 2012:8:528-33.

20. Reimer D, Cowles KN, Proschak A, Nollmann Fl, Dowling AJ, Kaiser M, French-Constant R, Goodrich-Blair H, Bode HB. Rhabdopeptides as insectspecific virulence factors from entomopathogenic bacteria. ChemBioChem. 2013;14:1991-7.

21. Houard J, Aumelas A, Noël T, Pages S, Givaudan A, Fitton-Ouhabi V, VillainGuillot P, Gualtieri M. Cabanillasin, a new antifungal metabolite, produced by entomopathogenic Xenorhabdus cabanillasii JM26. J Antibiot (Tokyo). 2013;66:617-20
22. Singh J, Banerjee N. Transcriptional analysis and functional characterization of a gene pair encoding iron-regulated xenocin and immunity proteins of Xenorhabdus nematophila. J Bacteriol. 2008;190:3877-85.

23. Thaler JO, Baghdiguian S, Boemare N. Purification and characterization of xenorhabdicin, a phage tail-like bacteriocin, from the lysogenic strain F1 of Xenorhabdus nematophilus. Appl Environ Microbiol. 1995;61:2049-52.

24. Boemare NE, Boyer-Giglio MH, Thaler JO, Akhurst RJ, Brehelin M. Lysogeny and bacteriocinogeny in Xenorhabdus nematophilus and other Xenorhabdus spp. Appl Environ Microbiol. 1992;58:3032-7.

25. Fuchs SW, Proschak A, Jaskolla TW, Karas M, Bode HB. Structure elucidation and biosynthesis of lysine-rich cyclic peptides in Xenorhabdus nematophila. Org Biomol Chem. 2011;9:3130-2.

26. Malan AP, Knoetze R, Moore SD. Isolation and identification of entomopathogenic nematodes from citrus orchards in South Africa and their biocontrol potential against false codling moth. J Invertebr Pathol. 2011;108:115-25

27. de Waal JY, Malan AP, Addison MF. Efficacy of entomopathogenic nematodes (Rhabditida: Heterorhabditidae and Steinernematidae) against codling moth, Cydia pomonella (Lepidoptera: Tortricidae) in temperate regions. Biocontrol Sci Tech. 2011:21:1161-76.

28. le Vieux PD, Malan AP. The potential use of entomopathogenic nematodes to control Planococcus ficus (Signoret) (Hemiptera: Pseudococcidae). South African J Enol Vitic. 2013;34:296-306.

29. Pillay U, Martin LA, Rutherford RS, Berry SD. Entomopathogenic nematodes in sugarcane in South Africa. Proc South African Sugar Technol Assoc. 2009; 82:538-41.

30. Malan AP, Manrakhan A. Susceptibility of the Mediterranean fruit fly (Ceratitis capitata) and the Natal fruit fly (Ceratitis rosa) to entomopathogenic nematodes. J Invertebr Pathol. 2009;100:47-9.

31. Webster JM, Li J, Chen G. Indole derivatives with antibacterial and antimycotic properties. US5569668A. 1995. Canada.

32. Gregson RP, Mclnerney B V. Xenocoumacins. EP0192713B1. 1991. Australia.

33. Webster JM, Li J, Chen G. Xenomins novel heterocyclic compounds with antimicrobial and antneoplastic properties. US5827872A. 1998. Canada.

34. Webster JM, Li J, Chen G. Heterocyclic compounds with antibacterial and antimycotic properties. US6316476B1. 2001. Canada.

35. Rhodes SH, Lyons GR, Gregson RP, Akhurst RJ, Lacey MJ. Xenorhabdin antibiotics. WO1984001775A1. 1984. Australia.

36. Gaultieri M, Villain-Guillot P, Givaudan A, Pages S. Cabanillasin, a new antifungal compound, produced by entomopathogenic Xenorhabdus cabanillasii. EP2468718A1. 2012. France.

37. Gaultieri M, Villain-Guillot P, Givaudan A, Pages S. Nemaucin, an antibiotic produced by entomopathogenic Xenorhabdus cabanillasii. WO2012085177 A1. 2012. France.

38. Weber T, Blin K, Duddela S, Krug D, Kim HU, Bruccoleri R, Lee SY, Fischbach MA, Müller R, Wohlleben W, Breitling R, Takano E, Medema MH. AntiSMASH 3.0 - a comprehensive resource for the genome mining of biosynthetic gene clusters. Nucleic Acids Res. 2015;43:W237-43.

39. Cimermancic P, Medema MH, Claesen J, Kurita K, Wieland Brown LC, Mavrommatis K, Pati A, Godfrey PA, Koehrsen M, Clardy J, Birren BW, Takano E, Sali A, Linington RG, Fischbach MA. Insights into secondary metabolism from a global analysis of prokaryotic biosynthetic gene clusters. Cell. 2014; 158:412-21

40. Rautenbach M, Vlok NM, Eyéghé-Bickong HA, Van der Merwe MJ, Stander MA. An electrospray mass spectrometry study on the "in vacuo" heterooligomers formed by the antimicrobial peptides, surfactin and gramicidin S. Am Soc Mass Spectrom. 2017;28:1623-37.

41. Dreyer J, Malan AP, Dicks LMT. Three novel Xenorhabdus-Steinernema associations and evidence of strains of Xenorhabdus khoisanae switching between different clades. Current Microbiol. 2017;74:938-42.

\section{Publisher's Note}

Springer Nature remains neutral with regard to jurisdictional claims in published maps and institutional affiliations. 